\title{
Structural and biomechanical basis of mitochondrial movement in eukaryotic cells
}

\author{
This article was published in the following Dove Press journal: \\ International Journal of Nanomedicine \\ 23 October 2013 \\ Number of times this article has been viewed
}

Min Wu'

Aruna Kalyanasundaram ${ }^{2}$

jie Zhu'

'Laboratory of Biomechanics and Engineering, Institute of Biophysics, College of Science, Northwest A\&F University, Yangling, Shaanxi, People's Republic of China; ${ }^{2}$ College of Pharmacology, University of Illinois at Chicago, Chicago, IL, USA
Correspondence: Jie Zhu Laboratory of Biomechanics and Engineering, Institute of Biophysics, College of Science, Northwest A\&F University, Yangling, Shaanxi 7I2100, People's Republic of China

Tel +86 2987084365

Fax +86 2987084365

Email jiezhu@nwafu.edu.cn

\begin{abstract}
Mitochondria serve as energy-producing organelles in eukaryotic cells. In addition to providing the energy supply for cells, the mitochondria are also involved in other processes, such as proliferation, differentiation, information transfer, and apoptosis, and play an important role in regulation of cell growth and the cell cycle. In order to achieve these functions, the mitochondria need to move to the corresponding location. Therefore, mitochondrial movement has a crucial role in normal physiologic activity, and any mitochondrial movement disorder will cause irreparable damage to the organism. For example, recent studies have shown that abnormal movement of the mitochondria is likely to be the reason for Charcot-Marie-Tooth disease, amyotrophic lateral sclerosis, Alzheimer's disease, Huntington's disease, Parkinson's disease, and schizophrenia. So, in the cell, especially in the particular polarized cell, the appropriate distribution of mitochondria is crucial to the function and survival of the cell. Mitochondrial movement is mainly associated with the cytoskeleton and related proteins. However, those components play different roles according to cell type. In this paper, we summarize the structural basis of mitochondrial movement, including microtubules, actin filaments, motor proteins, and adaptin, and review studies of the biomechanical mechanisms of mitochondrial movement in different types of cells.
\end{abstract}

Keywords: mitochondrial movement, microtubules, actin filaments, motor proteins, adaptin

\section{Introduction}

The movement of mitochondria, which serves to distribute mitochondrial functions in a dynamic and nonuniform fashion, has attracted particular interest in recent years following the discovery of functional connections between microtubules, motor proteins, and the mitochondria, and their influence in neurodegenerative disease. ${ }^{1}$ Mitochondrial movements are able to be directed by the various components of the cytoskeleton, including actin filaments, microtubules, intermediate filaments, and other proteins. ${ }^{2}$ Many studies have shown that both actin filaments and microtubules play a part in mitochondrial movement, although the contribution of each cytoskeletal element changes considerably depending on specific organisms and cells. In most animal cells and some algae, mitochondrial movements are associated with microtubules and motor proteins. ${ }^{3-6}$ Studies in insect cells ${ }^{7}$ and neuronal axons ${ }^{8}$ have revealed that actin filaments can also serve as tracks for mitochondrial transport.

In higher plants, it has been shown that the mitochondria are closely associated with actin filaments, and mitochondrial movement is mainly dependent on intact actin filaments. ${ }^{9-11}$ In addition, in microorganisms, particularly in yeast, mitochondrial movement depends on actin cables instead of microtubules. Actin cables are composed 
of bundles of short actin filaments, ${ }^{12}$ and these undergo continuous turnover in yeast. ${ }^{13}$ Qualitatively similar actin dynamics have also been reported in plant cells, where single actin filaments are more dynamic than actin cables. ${ }^{14}$ Motor proteins and adaptor proteins are essential in the antegrade or retrograde movement of mitochondria. ${ }^{9}{ }^{9}$ Here we review our current understanding of the regulatory mechanisms that govern mitochondrial movement to supply some theoretical foundation for treatment of neurodegenerative diseases and to offer an explanation for the mechanisms controlling organelle inheritance. In most studies of mitochondrial movement in animals and microorganisms, budding yeast and nerve cells are the research subjects, respectively, and studies on mitochondrial movement of plants mainly concentrate on pollen cells and root hair cells. As the representatives of mitochondrial movement patterns in microorganisms, animals, and plants, respectively, these materials are typical for most eukaryotic cells' basic mechanisms for mitochondial movement. In this paper, the structural and biomechanical basis of mitochondrial movements of budding yeast, neuronal axons, and higher plants are individually summarized.

\section{Budding yeast}

Budding yeast, ie, Saccharomyces cerevisiae, is an anaerobe. If there is sufficient oxygen, facultative anaerobes get energy from mitochondrial aerobic respiration; if not, they can obtain energy by fermentation or anaerobic respiration. S. cerevisiae can tolerate mitochondrial mutations that would be lethal in other organisms. Therefore, budding yeast has been used widely to study the mechanisms of organelle inheritance. ${ }^{16-20}$ As a result, much of what we know regarding mitochondrial movement has come from using budding yeast as a model system. In budding yeast, cytoplasm microtubules are sparse, mitochondrial movement is not dependent on microtubules, and forces for movement are generated by Arp2/3 complexstimulated actin polymerization. ${ }^{21-24}$ Mitochondria would stop moving if they were treated by drugs that destroy the actin cytoskeleton, remove actin-binding protein, or change the myosin binding sites on actin monomers. Photographic images of actin cytoskeleton synchronization in yeast mitochondria show that the mitochondria move along actin protein cables. ${ }^{15}$

Mitochondrial transport from mother to daughter buds during asymmetric cell division in budding yeast ensures that the healthiest organelles are inherited by the new generation. This inheritance is critical and is mediated by type $\mathrm{V}$ myosin motor Myo2, which transports mitochondrial membranes along actin cables into the bud. ${ }^{25,26}$ Two additional proteins, Ypt11 and Mmr1, interact with the cargo-binding domain on the Myo2 tail and participate in mitochondrial inheritance. ${ }^{27,28}$ A recent study using live cell microscopy revealed that Num1 is required for attachment of mitochondria to the cell cortex and retention in mother cells, and that expression of chimeric plasma membrane tethers rescued mitochondrial fission defects in $\Delta$ num 1 and $\Delta \mathrm{mdm} 36$ mutants. ${ }^{20}$

The pattern of mitochondrial distribution is closely linked to the cell cycle, and is demonstrated by time-lapse imaging of mitochondria in the cell cycle of budding yeast. ${ }^{22}$ Mitochondria are equally distributed between the daughter and mother cell prior to cell-cell separation. With improved spatial and temporal resolution of 4D imaging (time lapse imaging combined with $3 \mathrm{D}$ reconstruction), it was discovered that mitochondria undergo motility events that are more rapid and distributive than previously thought. ${ }^{15}$ It was also found that mitochondria undergo rapid, bi-directional movement in budding yeast. ${ }^{15}$ Budding is an important process in yeast cells, which produces more cells. Before budding, the mitochondria are arranged along the mother-daughter axis and converge to the future budding site. Mitochondrial movement into the bud occurs as soon as the bud emerges, while mitochondria in the mother cell also show retrograde movement to ensure a balanced distribution of mitochondria in the mother cell and daughter cells. Therefore, this balance is related to three states of mitochondrial movement, ie, antegrade movement, retrograde movement, and reservations at the ends.

\section{Retrograde movement of mitochondria in budding yeast}

Observation reveals that actin cables in yeast are in a state of constant movement, which is elongated in yeast budding. ${ }^{29}$ Using Abp140p-GFP to study actin cable dynamics, it was found that elongating actin cables move in a retrograde direction from the bud towards the mother cell at a rate of $0.3 \mu \mathrm{m}$ per second. Actin cable elongation occurs by insertion of newly polymerized proteins into the end of the actin cable at the actin cable assembly site. The main proteins involved are F-actin, resident actin cable proteins, tropomyosin proteins (Tpm1/2p), and actin bundling proteins (Abp140p and Sac6p). ${ }^{30}$ These insertions happen during elongation of the actin cables (see Figure 1). Thus, the velocity of retrograde mitochondrial movement is similar to that of the associated retrograde actin cable flow. Mitochondria move with the retrograde flow of actin cables. Actin cable elongation occurs by insertion of newly polymerized F-actin and resident actin cable proteins, ie, tropomyosin proteins (Tpm1/2p) and actin bundling proteins (Abp140p and Sac6p), into the end of the actin cable at the actin cable assembly site. This insertion results in movement 


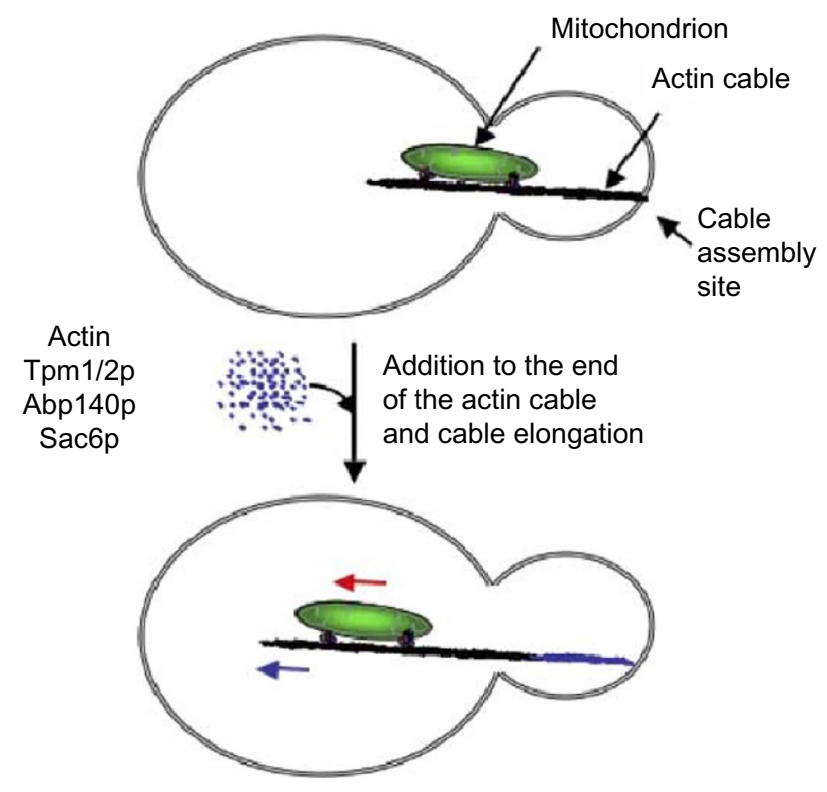

Figure I Sketch model for retrograde mitochondrial movement in budding yeast. Note: Reprinted from Gene, 354, Boldogh IR, Fehrenbacher KL, Yang HC, Pon LA, Mitochondrial movement and inheritance in budding yeast, 28-36, copyright (2005), with permission from Elsevier. ${ }^{30}$

of the mitochondria along the elongating actin cable in the retrograde direction, ie, towards the mother cell.

\section{Antegrade mitochondrial movement}

During the budding process, mitochondria move in an antegrade manner to reach the daughter cells. This process requires not only the guidance of actin cables but also several mediator proteins. According to the model of antegrade mitochondrial movement, the mitochore complex (Mmm1p, Mdm10p, and $\mathrm{Mdm} 12 \mathrm{p}$ ) mediates cyclic binding of mitochondria to actin cables. At the same time, the Arp2/3 complex, which is recruited by the PUF protein, Jsn 1p, stimulates actin polymerization for generation of antegrade force. ${ }^{30}$ This complex works at the interface between the organelle and its cytoskeletal track. The forces produced by this process are enough to overcome the opposing force of retrograde actin cable flow, and allow for mitochondrial movement toward the bud end (see Figure 2).

When mitochondria associated with the mitochore have not recruited the Arp2/3 complex with the help of Jsn1p, they have to move back to the mother end with retrograde flow of the actin cable. However, if they have recruited the Arp2/3 complex, which can supply energy by promoting actin polymerization, the mitochondria can move in an antegrade fashion to the daughter end.

The mitochore complex participates in the connection between mitochondria and actins by recruiting Arp2/3. A defect in Mmm1p, Mdm10p, or Mdm12p will result in

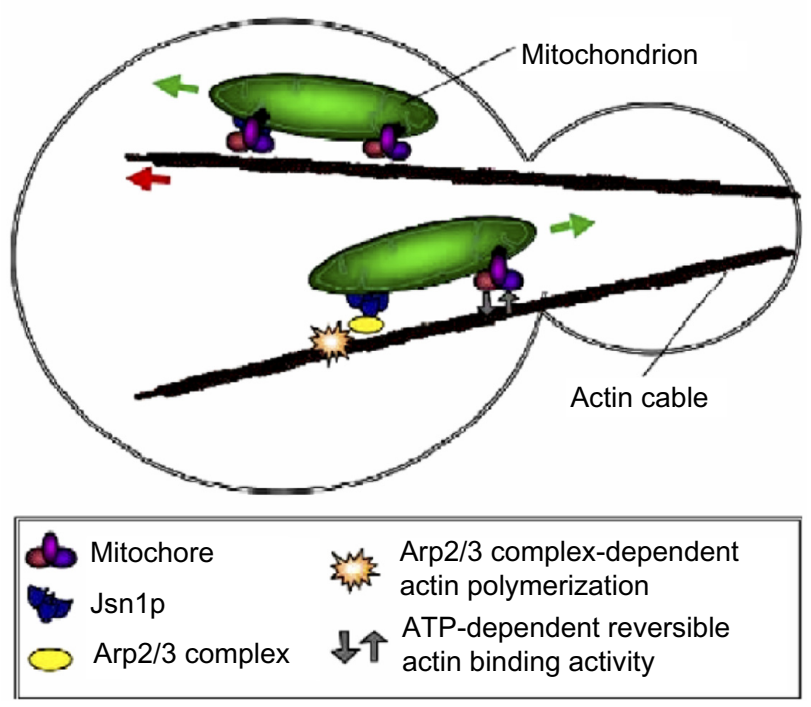

Figure 2 Model of mitochondria antegrade and retrograde movement in budding yeast. Note: Reprinted from Gene, 354, Boldogh IR, Fehrenbacher KL, Yang HC, Pon LA, Mitochondrial movement and inheritance in budding yeast, 28-36, copyright (2005), with permission from Elsevier. ${ }^{30}$

Abbreviation: ATP, adenosine triphosphate.

genetic deletion of mitochondria, variation in mitochondrial morphology, and defective B-barrel proteins in the outer membrane of the mitochondria. If there is a defect in Mdm10p, the mitochondria form a ball-like structure, which hinders the movement of mitochondia toward the bud cell. ${ }^{25}$ However, mutation of Mdm10p would not affect nuclear inheritance or disturb cell activity and function.

The Arp2/3 complex produces the power needed for antegrade movement of the mitochondria. Photographic images of living cells demonstrate that recruitment and activation of Arp2/3 is needed for antegrade mitochondrial movement. ${ }^{31,32}$ The Arp2/3 complex can join with F-actin, which is associated with formation of the actin network on the mitochondrial surface. The mitochondrion combines with the actin cable with the help of the mitochore. Jsn $1 p$ recruits the Arp2/3 complex to provide power for antegrade movement. The mitochondrion can then be transported to the bud instead of back to the mother cell as happens with retrograde flow. Peripheral mitochondrial proteins, such as Jsn1p, can connect the purified Arp2/3 subunit, and are necessary for antegrade mitochondrial movement. Therefore, Jsn $1 \mathrm{p}$ is likely to be the receptor protein for the Arp2/3 complex.

\section{Mitochondrial retention at the tips}

During division of budding yeast cells, the mitochondria first need to move from the mother cell to the bud; however, mitochondrial retention in the mother cell prevents all of the mitochondria being transported to the bud tips. 
Theoretically, retrograde flow of actin cables would bring the mitochondria back to the mother cells. Thus, it is necessary for mitochondria to be anchored at the tips.

Some studies support a role for type V myosin and its binding partner, Rab-like G-protein (Ypt11p), in anchorage of mitochondria in the bud tips. There are two forms of type $\mathrm{V}$ myosin in budding yeast, ie, Myo2p and Myo4p. Type V myosin can act as a motor that directs active transport of cargo along actin cables in yeast. In the mutant form, by changing Myo2 orYpt11, there are some defects of mitochondrial retention in the bud tips. However, deletion of Ypt11 or Myo2 has no effect on antegrade mitochondrial movement or colocalization of the mitochondria with actin cables. ${ }^{29}$ Thus, it is possible that Myo2p and Ypt1 1p drive transport of retention factors to the site of the bud tips (see Figure 3). Antegrade movement is powered by Myo2 bound to the mitochondria via an as yet unknown receptor protein (X). The black square in Figure 3 denotes hypothetical mitochondrial cortex anchors.

\section{Motor-dependent mechanism of mitochondrial movement}

There is another possible path way for mitochondrial movement, whereby the mitochondria rely on connection to actin cables by a type of motor instead of the mitochore. For example, Myo2p could participate in the transportation of some organelles, and peripherins, such as Mmrlp and Ypt11p, can combine with Myo2p. Their overexpression can drive the mitochondria to move toward the bud tips. There is some evidence that Myo2p can move along the actin cables as a mitochondrial motor protein, with Mmr1p or Ypt11p acting as its adaptor proteins. ${ }^{33}$

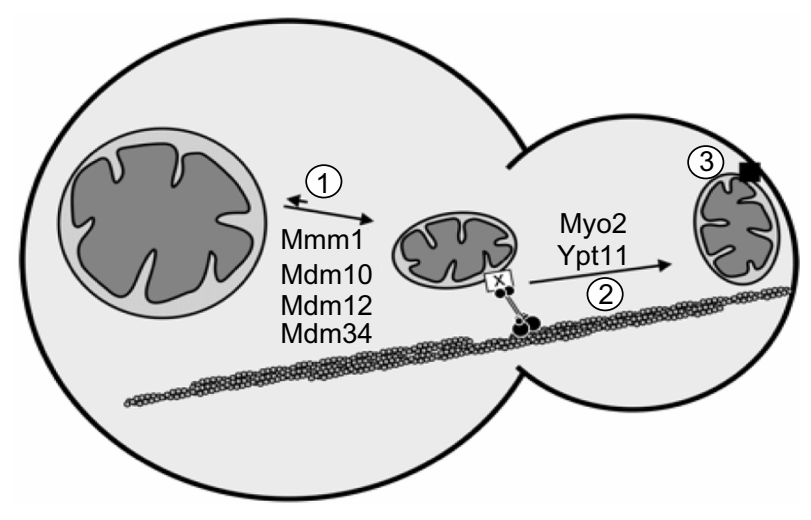

(1) Maintenance of transportable mitochondria units

(2) Bud-directed mitochondrial transport

(3) Retention of mitochondrial at the bud tip

Figure 3 Model of mitochondrial retention.

Note: Reprinted with permission from (C) 201 I Förtsch et al. Journal of Cell Biology. 194:473-488. doi:10.1083/jcb.201012088. ${ }^{25}$

\section{Nerve cells}

Neurons are particularly susceptible to disturbance of mitochondrial motility and distribution due to their highly extended structures and specialized function. ${ }^{34}$ Regulation of mitochondrial motility plays a vital role in neuronal health and death. Experimental evidence points to the importance of mitochondrial movement defects as contributors to major neurodegenerative diseases, such as Parkinson's disease. ${ }^{35}$ Recent research shows that two Parkinson's disease proteins, Ser/Thr kinase (PINK1) and ubiquitin ligase (Parkin), participate in this regulation by arresting mitochondrial movement. ${ }^{36}$ This study provides important clues as to the mechanisms of Parkinson's disease, and strengthens the relationship between mitochondrial movement and brain damage. As more mitochondrial movement-related protein is analyzed, we will understand these mechanisms better and be able to provide useful information for the treatment of neurodegenerative disease.

The neuron is a highly differentiated cell consisting of three distinct functional domains, ie, a cell body, a long axon, and a dendrite. The distribution of mitochondria in nerve cells is aligned with the level of cellular energy and the demand of metabolism. The extreme length of the axonal processes requires that aerobic adenosine triphosphate (ATP) production and $\mathrm{Ca}^{2+}$ homeostasis are nonuniformly organized in the cytoplasm. As a result, mitochondrial movement along axons is essential for neuronal homeostasis. Furthermore, mitochondrial starts, stops, and redistributions can be regulated by physiologic events and intracellular signals. Mitochondria undergo rapid but intermittent transport in both the antegrade and retrograde directions in axons.

Mitochondria with a high potential have been shown to move towards and accumulate in the growth cone, while mitochondria with a low potential are transported towards the cell body, which increases the possibility of antegrade movement of mitochondria to the sites of action of neurons, and retrograde movement to the cell body for decomposition and recycling. ${ }^{37}$

\section{Role of cytoskeleton in mitochondrial movement}

The cytoskeleton not only provides structural support for neurons but also supplies the conditions for transport and retention of organelles. Eukaryotic cells contain three main kinds of cytoskeletal filaments, ie, actin filaments, intermediate filaments, and microtubules. Actin filaments and microtubules have both plus and minus ends and are polarized, whereas the intermediate filaments do not have plus and minus ends. 
Microtubules are formed by polymerization of a dimer of two globular proteins, ie, alpha and beta tubulin. Many proteins bind to microtubules, including motor proteins such as kinesin, dynein, and katanin, as well as other proteins important for regulating microtubule dynamics. The directions of microtubule distribution are not the same in all parts of the neuron. Microtubules always run longitudinally and act as rails for transport in both axons and dendrites. In the axon, microtubules are polarized and arranged in parallel, with the positive terminal pointing towards the synapse. However, in dendrites, microtubules are arranged in parallel and antiparallel directions. ${ }^{38}$ This structure and arrangement of microtubules is very important for the combination between motor proteins and mitochondrial movement. Generally, kinesin mediates mitochondrial movement along the axon in an antegrade direction, and dynein would be involved in the relevant retrograde processing.

Actin filaments are helical polymers composed of actin monomers, the two ends of which grow at different rates. As the thinnest filaments of the cytoskeleton, actin filaments are composed of linear polymers of actin subunits, and generate force by elongation at one end of the filament coupled with shrinkage at the other, causing net movement of the intervening strand. In nerve cells, actin filaments distribute to the parts with fewer microtubules (such as the presynaptic terminal and dendritic spines), and are very important for the mitochondria during short distance transportation. In neurons, actin fibers can also form a reticulate structure which directly influences myosin activity. There are some mitochondrial movementrelated motor proteins along the actin filaments. Indirect evidence suggests that the myosin I, II, V, VI family could be the motor proteins enabling the mitochondria to move along the actin filament, although the specific mechanism involved has not yet been identified. ${ }^{2}$

The intermediate filaments, averaging $10 \mathrm{~nm}$ in diameter, are more stable (strongly bound) than actin filaments, and are heterogeneous constituents of the cytoskeleton. Like actin filaments, they function in the maintenance of cell shape by bearing tension. (Microtubules, by contrast, resist compression. It may be useful to think of micro- and intermediate filaments as cables, and of microtubules as cellular support beam.) These filaments provide potential binding sites for the mitochondria and other proteins. There is some evidence for an interaction between the intermediate filaments and mitochondria. For example, it has been proved that vimentin intermediate filaments join the mitochondrial binding with the N-end and inhibit mitochondrial movement. ${ }^{39}$ Being an intermediate filament protein, desmin can affect distribution of mitochondria in the striated muscle cell, but inhibits mitochondrial recruitment of kinesin in the cardiac muscle cell. ${ }^{40}$

\section{Motor proteins involved in mitochondrial movement}

Motor proteins can transport membranous organelles and macromolecules fundamental for cellular function along microtubules, and the transporting roles of these proteins have been studied extensively in axons and dendrites. Recent findings have revealed that the specific interaction between cargoes and motors is mediated in many cases via adaptor proteins. Cargoes could be sorted into precise destinations by these motors, such as axons or dendrites. Long distance transport of mitochondria toward the axon is along microtubules, and depends on motor proteins powered by ATP from mitochondrial ATP synthase. Kinesin mediates antegrade mitochondrial movement along both the axon and dendrite, and dynein would be involved in the retrograde transportation.

Six members of the kinesin family (ie, kinesin-1, 2, 3, 4,13 , and 14) have been implicated in axonal organelle transport, with two of them particularly involved in mitochondrial movement. The widely expressed "conventional" kinesins of the kinesin-1 family are associated with the mitochondria as well as other axonal organelles, such as vesicles containing ApoER2, JNK scaffolding proteins, and amyloid precursor protein. ${ }^{41-43}$ The founding member of this superfamily, kinesin-1, was isolated as a heterotetrameric fast axonal organelle transport motor consisting of two identical motor subunits (KFCs; kinesin heavy chains) and two "light chains" (kinesin light chain) via microtubule affinity purification from neuronal cell extracts. The heavy chains of kinesin-1, also known as KIF5, can function as a motor and form homodimers or heterodimers among themselves through the coiled-coil region in the stalk domains. Each KIF5 heavy chain contains an N-terminal motor domain that binds directly to microtubules, whereas its C-terminal domain mediates the association of KIF5 with kinesin light chains or direct interaction with intracellular cargo vesicles. Therefore, attachment of kinesin-1 can transport directly through the specific cargo-binding region at the C-terminal domain, or indirectly via the light chains, indicating two distinct forms of motor-cargo coupling (see Figure 4). Biochemical and genetic evidence demonstrates that recruitment of kinesin heavy chain KIF5 and mitochondrial movement are independent of kinesin light chains, which inhibit KIF5 binding to Milton. ${ }^{44}$ 


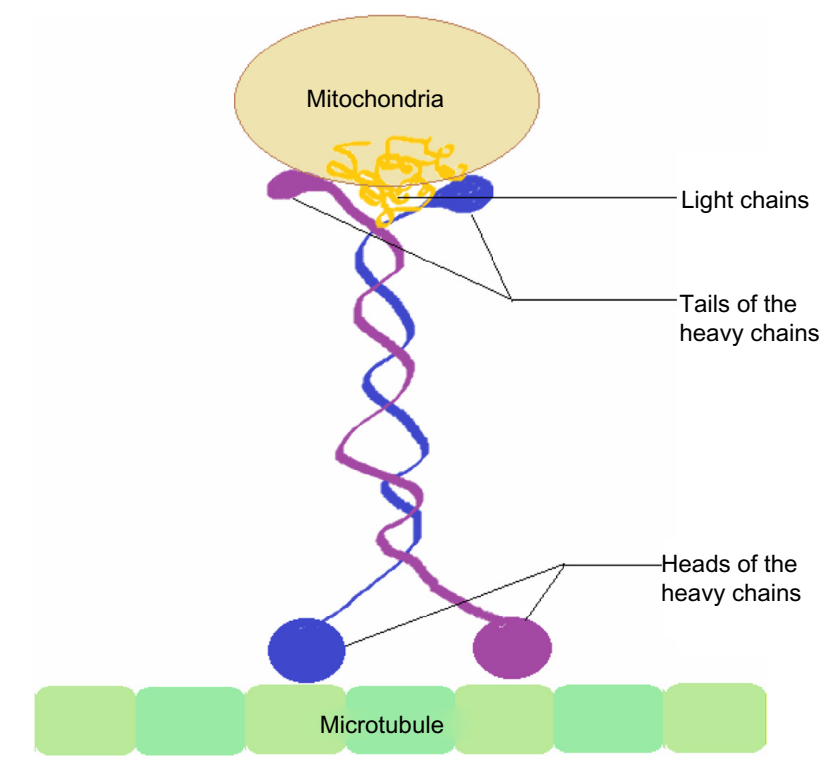

Figure 4 Model of mitochondrial movement driven by kinesin along a microtubule.

The kinesin heavy chain includes the heads, tails, and necks that connect the heads to the tails. The head is the signature of kinesin and its amino acid sequence is well conserved among various kinesins. Each head has two separate binding sites, ie, one for the microtubule and the other for ATP. ATP binding and hydrolysis as well as ADP release change the conformation of the microtubule-binding domains and the orientation of the neck linker with respect to the head, resulting in the motion of kinesin.

Dyneins can be divided into two groups, ie, cytoplasmic dyneins and axonemal dyneins, which are also called ciliary or flagellar dyneins. Cytoplasmic dynein, which has a molecular mass of about $1.5 \mathrm{mDa}$, contains approximately 12 polypeptide subunits: two identical "heavy chains," $520 \mathrm{kDa}$ in mass, which contain ATPase activity and are thus responsible for generating movement along the microtubule; two $74 \mathrm{kDa}$ intermediate chains which are believed to anchor the dynein to its cargo; and four 53-59 kDa intermediate chains and several light chains which are less well understood (see Figure 5) at this moment. Axonemal dynein causes sliding of microtubules in the axonemes of cilia and flagella, and is found only in cells that have those structures. Axonemal dynein comes in multiple forms that contain either one, two, or three nonidentical heavy chains, depending on the organism and location in the cilium. Each heavy chain has a globular motor domain with a doughnut-shaped structure believed to resemble that of other proteins, a coiled coil "stalk" that binds to the microtubule, and an extended tail that attaches to a neighboring microtubule of the same axoneme. Cytoplasmic dynein is

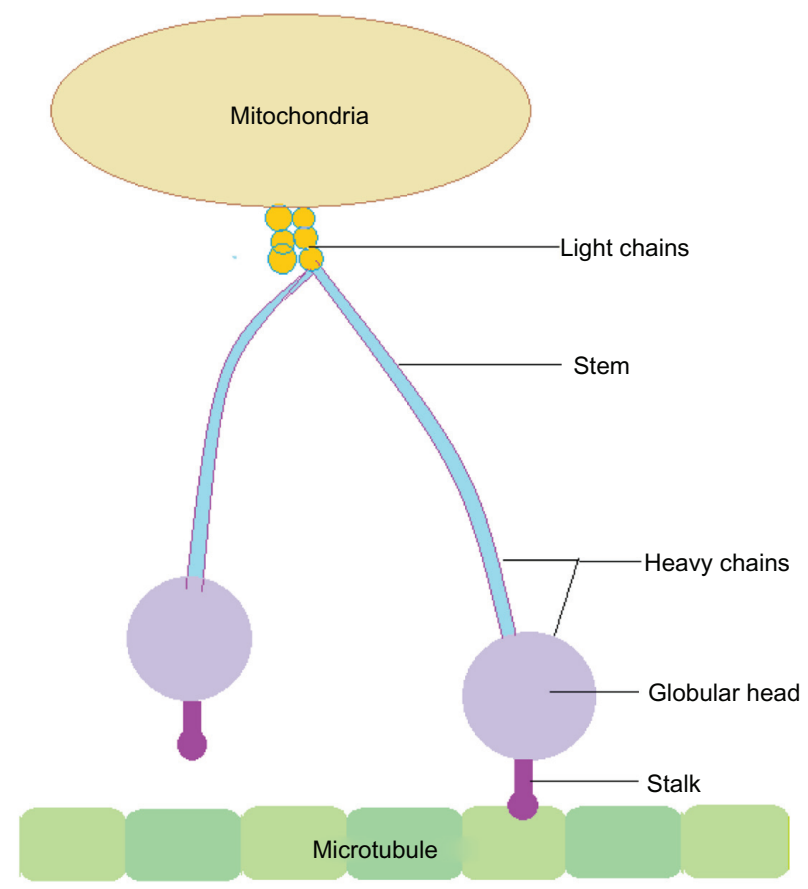

Figure 5 Model of mitochondrial movement driven by dynein along a microtubule.

essential for axonal transport of mitochondria in neurons. In Drosophila, cytoplasmic dynein is the primary motor for mediating mitochondrial retrograde transport. ${ }^{45}$ Studies of single dynein-dynactin motor complexes revealed that the complex could move bidirectionally, although the overall bias in direction is towards the microtubule minus ends. ${ }^{46,47}$

The two giant heads of dynein are made up of part of a heavy chain. The rest of the heavy chain and the intermediate light chain form the stem to bind heavy chain head and light chain tail. The light chain can associate with the cargo. Unlike kinesin, the heavy chain head does not connect with the microtubule directly; instead, it moves on the microtubule through a segment of stalk.

Neurons have multiple compartments that are devoid of microtubules where transport of organelles is still seen to occur. These areas are rich in other cytoskeletal polymers, such as actin filaments. Transported organelles have been shown to associate with multiple motor proteins including myosins, which suggests that nonmicrotubule-based transport may be a myosin-driven process. ${ }^{48}$ While the microtubulebased motor proteins, kinesin and dynein, quickly transport cargoes and organelles through lengthy axons, myosins drive short-distance trafficking along actin filaments at presynaptic terminals and growth cones. Most myosin molecules are composed of a head, neck, and tail domain. The head domain binds filamentous actin and uses ATP hydrolysis 
to generate force and "walk" along the filament towards the barbed (+) end (with the exception of myosin VI, which moves towards the pointed [-] end). The neck domain acts as a linker and as a lever arm for transducing force generated by the catalytic motor domain. The neck domain can also serve as a binding site for myosin light chains, which are distinct proteins forming part of a macromolecular complex and generally having regulatory functions. The tail domain generally mediates interaction with cargo molecules and/or other myosin subunits. In some cases, the tail domain may play a role in regulating motor activity. Although myosins I, II, V, and VI were reported to be involved in the transport of organelles and vesicles in neurons, with the exception of myosin V, little is known about the location and function of other unconventional myosins and their roles in axonal mitochondrial transport. Myosin V is a good candidate for driving actin-based mitochondrial movement, although it remains to be determined whether myosin $\mathrm{V}$ is attached to the axonal mitochondria and how much or to what degree synapse-directed transport of mitochondria is required for myosin $\mathrm{V}^{48,49}$ A study reveals that a novel unconventional myosin, Myo19, is a mitochondria-associated motor that may play a role in either the transport or tethering of this organelle. ${ }^{50,51}$

\section{Adaptors essential for mitochondrial movement}

Regarding neuronal mitochondria, the specificity of antegrade transport is achieved by direct interaction between KIF5s and the mitochondrial membrane or by indirect coupling via an adaptor that binds KIF5s and the mitochondria through lipid-binding or transmembrane domains. Several mitochondrial motor-adaptor complexes and receptors have been identified. ${ }^{52}$

Syntabulin was first identified in our laboratory and serves as another KIF5 motor adaptor that links KIF5 to syntaxin-containing vesicles. ${ }^{53}$ Syntabulin plays a critical role in mitochondrial trafficking in neurons. It is a peripheral membrane-associated protein that targets to mitochondria through its carboxyl-terminal tail. Syntabulin is a peripheral membrane-associated protein, relatively enriched in the mitochondrial fraction, and associated with mitochondria via its sequence. Immunocytochemical and time-lapse imaging studies in live neurons demonstrated that syntabulin colocalized and comigrated with mitochondria within axonal processes. Syntabulin serves as a KIF5 adaptor and mediates antegrade transport of mitochondria along neuronal processes..$^{54,55}$
Milton, a mitochondrial motor adaptor, provides a link between kinesin motors and mitochondria through an interaction with Miro, a calcium-sensing member of the Rho-GTPase family present in the outer mitochondrial membrane. ${ }^{56}$ The N-terminal of Milton is responsible for recruiting kinesin heavy chain to the mitochondria. Mutation of Milton will lead to loss of mitochondria in the synaptic terminal and axon. ${ }^{41}$ The Miro-Milton complex, as an adaptor complex containing three components at least, links kinesin to the mitochondrion. Milton links with kinesin heavy chain, Miro mediates Milton and the mitochondrion, while PINK1 localizes on the mitochondrial outer membrane by interacting with Miro and Milton (see Figure 6). ${ }^{57}$ Both Milton and syntabulin are important adaptins between the mitochondria and motor proteins, but work via different mechanisms, including the association with kinesin, the combination with mitochondria, the reaction to calcium signaling, and regulation of protein phosphorylation.

\section{Higher plant cells}

Strategic control of mitochondrial movements and cellular distribution is essential for correct plant cell function and survival. Despite being a vital process, mitochondrial movement in plant cells is a poorly documented phenomenon. Picea wilsonii pollen tubes and Arabidopsis root hairs have been used to investigate the role of actin filaments and microtubules in mitochondrial movement. ${ }^{58}$

Mitochondria can move rapidly along actin filaments over long distances in higher plants. ${ }^{9}$ In angiosperms, pollen tubes show a strong cytoplasmic streaming of organelles, which move along actin filaments, as shown by earlier studies with cytoskeleton inhibitors. ${ }^{59,60}$ Although less well supported, it is evident that microtubules also participate in regulation of

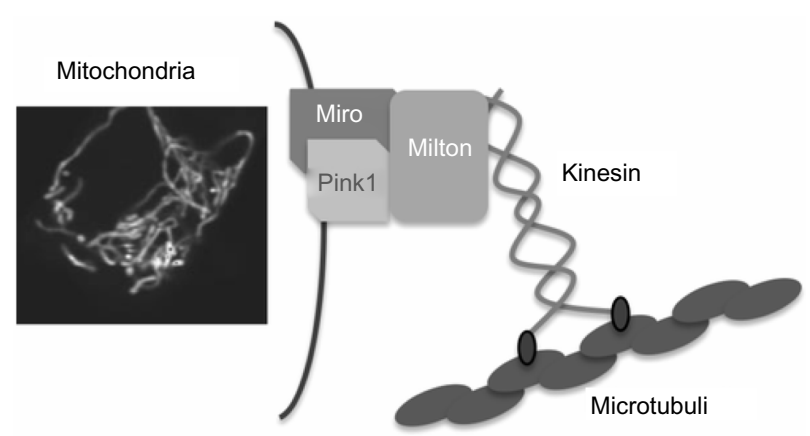

Figure 6 Model of the Miro-Milton complex linking the kinesin heavy chain and the mitochondrion.

Note: Adapted with permission from Weihofen A, Thomas KJ, Ostaszewski BL Cookson MR, Selkoe DJ. PinkI forms a multiprotein complex with Miro and Milton, linking PinkI function to mitochondrial trafficking. Biochemistry. 2009;48: 2045-2052. Copyright (2009) American Chemical Society. ${ }^{57}$ 
mitochondrial movement in higher plants. In nonvascular plants, such as the alga Chara, both microtubules and actin filaments are involved in the transport and immobilization of mitochondria. ${ }^{61}$ Mitochondria from tobacco pollen tubes move slowly along microtubules but more rapidly along actin filaments, and these mitochondria are associated with a specific microtubule-dependent motor. A $170 \mathrm{kDa}$ myosin is bound to mitochondria and Golgi vesicles, while a $90 \mathrm{kDa}$ kinesin is associated with mitochondria but not with vesicles. Research also suggests that kinesin and myosin cooperate for the positioning of mitochondria in the pollen tube. ${ }^{11}$

The research on Arabidopsis root hairs showed that: myosin and actin turnover cooperates in driving linear mitochondrial movements; the transition of mitochondria from immobility to movement involves de novo actin polymerization and depolymerization; and microtubule dynamics have profound effects on mitochondrial velocity, trajectory, and positioning by directing the arrangement of actin filaments. ${ }^{10}$ Thus, these data can be combined to generate a testable model which will expand our insights into the molecular control of mitochondrial movement in plant cells.

\section{Conclusion}

Cells precisely regulate mitochondrial movement in order to balance energy needs and avoid cell death. Mitochondrial movement and retention is a very important and complex process, involving many molecular motors and adaptor proteins.

In budding yeast cells, there are three states of movement of the mitochondria, ie, retrograde movement, antegrade movement, and reservations at the ends. In retrograde movement, the mitochondria move with the retrograde flow of actin cables via the forces generated by actin polymerization. In antegrade movement, mitochondria move toward the bud end via the forces produced by Arp2/3 complexstimulated actin polymerization. Arp $2 / 3$ is recruited by Jsn $1 \mathrm{p}$, and the mitochore complex participates in cyclic binding of the mitochondria to actin cables. In mitochondrial retention, Ypt11p supports a role in anchorage of mitochondria to the bud tips. Myo2 is also essential in mitochondrial retention.

In nerve cells, mitochondria move mostly along microtubules. Actin filaments are very important for mitochondria during short-distance transportation. The intermediate filaments can supply potential binding sites for the mitochondria and other proteins. A lot of motor proteins also participate in mitochondrial movement. Kinesin mediates antegrade mitochondrial movement along the axon, and dynein would be involved in the retrograde movement. Adaptors such as syntabulin and Milton also play a critical role in mitochondrial movement in neurons.

In plant cells, there is some evidence to suggest that mitochondrial movement at low speeds in pollen tubes is driven by myosin, while high-speed and variable-speed movements are powered by both actin filament dynamics and myosin, with kinesin and myosin cooperating in positioning of the mitochondria.

These discoveries continue to advance our field, but more importantly, provide new directions that expand our conceptualization of a more global cellular role for this fascinating organelle. The future of mitochondrial research is bright, and the contributions made in this area will provide a touchstone for future work.

\section{Acknowledgments}

This project was supported by the National Science Foundation of China (11202170 to JZ), China Postdoctoral Foundation (2012T50823 to JZ), Scientific Foundation of Provincial Sports Bureau of Shaanxi (13001 to JZ), and Fundamental Research Funds for the Central Universities (QN2011123 to JZ). The authors would also like to acknowledge Prof Wang GD (Biophysics) and Prof Ouyang WQ (Veterinary Medicine) at Northwest A\&F University for their suggestions and support.

\section{Disclosure}

The authors report no conflicts of interest in this work.

\section{References}

1. Saxton WM, Hollenbeck PJ. The axonal transport of mitochondria. J Cell Sci. 2012;125 Pt 9:2095-2104.

2. Anesti V, Scorrano L. The relationship between mitochondrial shape and function and the cytoskeleton. Biochim Biophys Acta. 2006;1757: 692-699.

3. Yi M, Weaver D, Hajnoczky G. Control of mitochondrial motility and distribution by the calcium signal: a homeostatic circuit. $J$ Cell Biol. 2004;167:661-672.

4. Koonce MP, Euteneuer U, McDonald KL, Menzel D, Schliwa M. Cytoskeletal architecture and motility in a giant freshwater amoeba, Reticulomyxa. Cell Motil Cytoskeleton. 1986;6:521-533.

5. Menzel D, Elsner-Menzel C. Co-localization of particle transport with microtubules in cytoplasmic exudates of the siphonous green alga Bryopsis. Bot Acta. 1989;102:241-248.

6. Hirokawa N. Kinesin and dynein superfamily proteins and the mechanism of organelle transport. Science. 1998;279:519-526.

7. Sturmer K, Baumann O, Walz B. Actin-dependent light-induced translocation of mitochondria and ER cisternae in the photoreceptor cells of the locust Schistocerca gregaria. J Cell Sci. 1995;108:2273-2283.

8. Chada SR, Hollenbeck PJ. Mitochondrial movement and positioning in axons: the role of growth factor signaling. J Exp Biol. 2003;206: 1985-1992.

9. Doniwa Y, Arimura S, Tsutsumi N. Mitochondria use actin filaments as rails for fast translocation in Arabidopsis and tobacco cells. Plant Biotechnol. 2007;24:441-447. 
10. Zheng M, Beck M, Müller J, et al. Actin turnover is required for myosindependent mitochondrial movements in Arabidopsis root hairs. PLoS One. 2009;4:e5961.

11. Romagnoli S, Cai G, Faleri C, Yokota E, Shimmen T, Cresti M. Microtubule- and actin filament-dependent motors are distributed on pollen tube mitochondria and contribute differently to their movement. Plant Cell Physiol. 2007;48:345-361.

12. Kamasaki T, Arai R, Osumi M, Mabuchi I. Directionality of F-actin cables changes during the fission yeast cell cycle. Nat Cell Biol. 2005; 7:916-917.

13. Pollard TD, Blanchoin L, Mullins RD. Molecular mechanisms controlling actin filament dynamics in nonmuscle cells. Annu Rev Biophys Biomol Struct. 2000;29:545-576.

14. Staiger CJ, Sheahan MB, Khurana P, Wang X, McCurdy DW. Actin filament dynamics are dominated by rapid growth and severing activity in the Arabidopsis cortical array. J Cell Biol. 2009;184:269-280.

15. Fehrenbacher KL, Yang HC, Gay AC, Huckaba TM, Pon LA. Live cell imaging of mitochondrial movement along actin cables in budding yeast. Curr Biol. 2004;14:1996-2004.

16. Catlett NL, Weisman LS. Divide and multiply: organelle partitioning in yeast. Curr Opin Cell Biol. 2000;12:509-516.

17. Bretscher A. Polarized growth and organelle segregation in yeast: the tracks, motors, and receptors. J Cell Biol. 2003;160:811-816.

18. Merzet S, Hammermeister M, Altmann K, Dürr M, Westermann B. Molecular machinery of mitochondrial dynamics in yeast. J Biol Chem. 2007;388:917-926.

19. Frederick RL, Shaw JM. Moving mitochondria: establishing distribution of an essential organelle. Traffic. 2007;8:1668-1675.

20. Klecker T, Scholz D, Förtsch J, Westermann B. The yeast cell cortical protein Num1 integrates mitochondrial dynamics into cellular architecture. J Cell Sci. 2013;126 Pt 13:2924-2930.

21. Simon VR, Swayne TC, Pon LA. Actin-dependent mitochondrial motility in mitotic yeast and cell-free system identification of a motor activity on the mitochondrial surface. J Cell Biol. 1995;130:345-354.

22. Simon VR, Karmon SL, Pon LA. Mitochondrial inheritance cell cycle and actin cable dependence of polarized mitochondrial movements in Saccharomyces cerevisiae. Cell Motil Cytoskeleton. 1997;37: 199-210.

23. Boldogh IR, Yang HC, Nowakowski WD, et al. Arp2/3 complex and actin dynamics are required for actin-based mitochondrial motility in yeast. Proc Natl Acad Sci US A. 2001;98:3162-3167.

24. Boldogh IR, Ramcharan SL, Yang HC, Pon LA. type V myosin (Myo2p) and a Rab-like G-protein (Ypt11p) are required for retention of newly inherited mitochondria in yeast cells during division. Mol Biol Cell. 2004;15:3994-4002.

25. Förtsch J, Hummel E, Krist M, Westermann B. The myosin-related motor protein Myo2 is an essential mediator of bud-directed mitochondrial movement in yeast. J Cell Biol. 2011;194:473-488.

26. Altmann K, Frank M, Neumann D, Jakobs S, Westermann B. The class $\mathrm{V}$ myosin motor protein, Myo2, plays a major role in mitochondrial motility in Saccharomyces cerevisiae. J Cell Biol. 2008;181: $119-130$

27. Itoh T, Toh-E A, Matsui Y. Mmrlp is a mitochondrial factor for Myo2p-dependent inheritance of mitochondria in the budding yeast EMBO J. 2004;23:2520-2530.

28. Eves PT, Jin Y, Brunner M, Weisman LS. Overlap of cargo binding sites on myosin $\mathrm{V}$ coordinates the inheritance of diverse cargoes. J Cell Biol. 2012;198:69-85.

29. Yang HC, Pon LA. Actin cable dynamics in budding yeast. Proc Natl Acad Sci U S A. 2002;99:751-756.

30. Boldogh IR, Fehrenbacher KL, Yang HC, Pon LA. Mitochondrial movement and inheritance in budding yeast. Gene. 2005;354: 28-36.

31. Huckaba TM, Gay AC, Pantalena LF, Yang HC, Pon LA. Live cell imaging of the assembly, disassembly, and actin cable-dependent movement of endosomes and actin patches in the budding yeast, Saccharomyces cerevisiae. J Cell Biol. 2004;167:519-530.
32. Kaksonen M, Sun Y, Drubin DG. A pathway for association of receptors, adaptors, and actin during endocytic internalization. Cell. 2003;115: 475-487.

33. Frederick RL, Okamoto K, Shaw JM. Multiple pathways influence mitochondrial inheritance in budding yeast. Genetics. 2008;178:825-837.

34. Lovas JR, Wang X. The meaning of mitochondrial movement to a neuron's life. Biochim Biophys Acta. 2013;1833:184-194.

35. Lu X, Kim-Han JS, O’Malley KL, Sakiyama-Elbert SE. Microdevice platform for visualizing mitochondrial transport in aligned dopaminergic axons. J Neurosci Methods. 2012;209:35-39.

36. Wang XN, Winter D, Ashrafi G, et al. PINK1 and Parkin target Miro for phosphorylation and degradation to arrest mitochondrial motility. Cell. 2011;147:893-906.

37. Peno HB, Lee CW. Mitochondrial clustering at the vertebrate neuromuscular junction during presynaptic differentiation J Neurobiol. 2006;66:522-536.

38. Hirokawa N, Takemura R. Kinesin superfamily proteins and their various functions and dynamics. Exp Cell Res. 2004;301:50-59.

39. Nekrasova OE, Mendez MG, Chernoivanenko IS, et al. Vimentin intermediate filaments modulate the motility of mitochondria. $\mathrm{Mol} \mathrm{Biol}$ Cell. 2011;22:2282-2289.

40. Boldogh IR, Pon LA. Mitochondria on the move. Trends Cell Biol. 2007; 17:502-510.

41. Horiuchi D, Barkus RV, Pilling AD, Gassman A, Saxton WM. APLIP1, a kinesin-binding JNK scaffold protein, influences bidirectional transport of vesicles and retrograde transport of mitochondria in Drosophila axons. Curr Biol. 2005;15:2137-2141.

42. Inomata H, Nakamura Y, Hayakawa A, et al. A scaffold protein JIP$1 \mathrm{~b}$ enhances amyloid precursor protein phosphorylation by JNK and its association with kinesin light chain. J Biol Chem. 2003;278: 22946-22955

43. Matsuda S, Matsuda Y, D’Adamio L. Amyloid beta protein precursor (AbetaPP), but not AbetaPP-like protein 2, is bridged to the kinesin light chain by the scaffold protein JNK-interacting protein 1. J Biol Chem. 2003;278:38601-38606.

44. Glater EE, Megeath LJ, Stowers RS, Schwarz TL. Axonal transport of mitochondria requires Milton to recruit kinesin heavy chain and is light chain independent. J Cell Biol. 2006;173:545-557.

45. Pilling AD, Horiuchi D, Lively CM, Saxton WM. Kinesin-1 and dynein are the primary motors for fast transport of mitochondria in Drosophila motor axons. Mol Biol Cell. 2006;17:2057-2068.

46. Mallik R, Petrov D, Lex SA, King SJ, Gross SP. Building complexity: an in vitro study of cytoplasmic dynein with in vivo implications. Curr Biol. 2005;15:2075-2085.

47. Ross JL, Wallace K, Shuman H, Goldman YE, Holzbaur EL. Processive bidirectional motion of dynein-dynactin complexes in vitro. Nat Cell Biol. 2006;8:562-570.

48. Bridgman PC. Myosin-dependent transport in neurons. J Neurobiol. 2004;58:164-174

49. Hollenbeck PJ. The pattern and mechanism of mitochondrial transport in axons. Front Biosci. 1996;10:d91-d102.

50. Margaret A, Titus MA. Motors: unleashing mitochondria. Curr Biol. 2009;19:R1076-R1078.

51. Quintero OA, Divito MM, Adikes RC, et al. Human Myo19 is a novel myosin that associates with mitochondria. Curr Biol. 2009;19: 2008-2013.

52. Cai Q, Davis ML, Sheng ZH. Regulation of axonal mitochondrial transport and its impact on synaptic transmission. Neurosci Res. 2011;70: 9-15.

53. Cai Q, Pan PY, Sheng ZH. Syntabulin-kinesin-1 family member 5B-mediated axonal transport contributes to activity-dependent presynaptic assembly. J Neurosci. 2007;27:7284-7296.

54. Cai Q, Gerwin C, Sheng ZH. Syntabulin-mediated anterograde transport of mitochondria along neuronal processes. J Cell Biol. 2005 170:959-969.

55. Cai Q, Sheng ZH. Mitochondrial transport and docking in axons. Exp Neurol. 2009;218:257-267. 
56. Saotome M, Safiulina D, Szabadkai G, et al. Bidirectional Ca2+dependent control of mitochondrial dynamics by the Miro GTPase. Proc Natl Acad Sci U S A. 2008;105:20728-20733.

57. Weihofen A, Thomas KJ, Ostaszewski BL, Cookson MR, Selkoe DJ. Pink1 forms a multiprotein complex with Miro and Milton, linking Pink1 function to mitochondrial trafficking. Biochemistry. 2009;48: 2045-2052.

58. Zheng M, Wang Q, Teng Y, et al. The speed of mitochondrial movement is regulated by the cytoskeleton and myosin in Picea wilsonii pollen tubes. Planta. 2010;231:779-791.
59. Mascarenhas JP, Lafountain J. Protoplasmic streaming, cytochalasin B, and growth of the pollen tube. Tissue Cell. 1972;4:11-14.

60. Lancelle SA, Hepler PK. Cytochalasin-induced ultrastructural alterations in Nicotiana pollen tubes. Protoplasma. 1988;2:65-75.

61. Foissner I. Microfilaments and microtubules control the shape, motility, and subcellular distribution of cortical mitochondria in characean internodal cells. Protoplasma. 2004;224:145-157.

\section{Publish your work in this journal}

The International Journal of Nanomedicine is an international, peerreviewed journal focusing on the application of nanotechnology in diagnostics, therapeutics, and drug delivery systems throughout the biomedical field. This journal is indexed on PubMed Central, MedLine, CAS, SciSearch $\AA$, Current Contents ${ }^{\circledR} /$ Clinical Medicine,
Journal Citation Reports/Science Edition, EMBase, Scopus and the Elsevier Bibliographic databases. The manuscript management system is completely online and includes a very quick and fair peer-review system, which is all easy to use. Visit http://www.dovepress.com/ testimonials.php to read real quotes from published authors. 